University of Wollongong

Research Online

Faculty of Engineering and Information

Faculty of Engineering and Information

Sciences - Papers: Part A

Sciences

$1-1-2016$

\title{
Young's modulus measurement using fibre-coupled self-mixing laser diode
}

Ke Lin

University of Wollongong, kl740@uowmail.edu.au

Yanguang Yu

University of Wollongong, yanguang@uow.edu.au

Jiangtao Xi

University of Wollongong, jiangtao@uow.edu.au

Qinghua Guo

University of Wollongong, qguo@uow.edu.au

Jun Tong

University of Wollongong, jtong@uow.edu.au

See next page for additional authors

Follow this and additional works at: https://ro.uow.edu.au/eispapers

Part of the Engineering Commons, and the Science and Technology Studies Commons

Research Online is the open access institutional repository for the University of Wollongong. For further information contact the UOW Library: research-pubs@uow.edu.au 


\title{
Young's modulus measurement using fibre-coupled self-mixing laser diode
}

\author{
Abstract \\ A new fibre-coupled self-mixing laser diode is developed for measurement of Young's modulus. Instead of \\ transmission in free space, the light path is coupled in a fibre, which enables the measuring system easily \\ and feasibly to be installed in many application cases. A specimen made of aluminium alloy 6061 is \\ tested by the system designed. The obtained value of Young's modulus is $70.02 \mathrm{GPa}$ with $0.16 \%$ \\ measurement accuracy, showing a high repeatability and a good agreement with the standard values \\ reported in literatures. \\ Keywords \\ laser, mixing, coupled, self, young, diode, modulus, measurement, fibre \\ Disciplines \\ Engineering | Science and Technology Studies \\ Publication Details \\ K. Lin, Y. Yu, J. Xi, Q. Guo, J. Tong \& H. Li, "Young's modulus measurement using fibre-coupled self-mixing \\ laser diode," in 2016 International Conference on Numerical Simulation of Optoelectronic Devices \\ (NUSOD), 2016, pp. 157-158.

\section{Authors} \\ Ke Lin, Yanguang Yu, Jiangtao Xi, Qinghua Guo, Jun Tong, and Hui Jun Li
}




\section{Young's modulus measurement using fibre-coupled self-mixing laser diode}

\author{
Ke Lin, Yanguang Yu, Jiangtao Xi, Qinghua Guo, Jun \\ Tong \\ School of Electrical, Computer and Telecommunications \\ Engineering \\ University of Wollongong, Northfields Avenue \\ Wollongong, Australia, NSW 2522
}

\author{
Email: yanguang@uow.edu.au \\ Huijun Li \\ School of Mechanical, Materials and Mechatronic \\ University of Wollongong, Northfields Avenue \\ Wollongong, Australia, NSW 2522
}

\begin{abstract}
A new fibre-coupled self-mixing laser diode is developed for measurement of Young's modulus. Instead of transmission in free space, the light path is coupled in a fibre, which enables the measuring system easily and feasibly to be installed in many application cases. A specimen made of aluminium alloy 6061 is tested by the system designed. The obtained value of Young's modulus is 70.02 GPa with $0.16 \%$ measurement accuracy, showing a high repeatability and a good agreement with the standard values reported in literatures.
\end{abstract}

\section{INTRODUCTION}

Young's modulus is one of most important parameters in material engineering and industrial design, especially for the development of new materials. It enters in almost every formula for calculation of other mechanical properties. Several approaches have been introduced for Young's modulus measurement since very early, such as tensile testing, four-bending test and indentation method. Recently, new techniques based on resonant methods are increasingly used for its simplicity, rapidness and high accuracy [1].

Previously we have exhibited a resonant method using impulse excitation technique based on self-mixing interferometry, experimentally showing the repeatability with high measurement accuracy[2]. This paper proposes a fibrecoupled self-mixing (FCSM) measuring method, which is more compact and invulnerable to noise and promising for a more potential application for multi-points detection in the future.

\section{PRINCIPLE}

Young's modulus (denoted by $E$ ) is connected with the geometry dimension of a specimen and its fundamental resonant frequency (denoted by $f_{R O}$ ). According to the standard released by ASTM E187621 [3], the Young's modulus of a regular rectangular specimen with known size can be calculated as below while $L / h \geq 20$ :

$$
E=0.9465 \cdot \frac{m f_{R O}{ }^{2} L^{3}}{b h^{3}} \cdot T
$$

where $L, b, h, m$ are respectively length, width, height and mass of the specimen for test, and $T=1+6.585(h / L)^{2}$.

The fiber-coupled self-mixing laser diode measuring system mainly consists of a LD and an external target to be tested as the specimen. The LD is at DC biased with the LD controller. The emitting laser from the LD is coupled into a coupler with a fiber, and it will be going out from a collimator at the other end, and then it will be focused onto one end of specimen. A small portion of the light will be back-scattered or reflected by the specimen and re-enter the fiber and then the LD internal cavity. Both the amplitude and frequency of the LD power are modulated by the movement of the specimen. This modulated LD power (denoted by $P(t)$ ) is referred to as an self-mixing interferometry (SMI) signal which is detected by the photodiode (PD) packaged in the rear of the LD and amplified by a trans-impedance amplifier, then recorded by an oscilloscope or collected by personal computer via data acquisition (DAQ) card.

The widely accepted mathematical model for an FCSMLD measuring system is presented in previous paper [2].

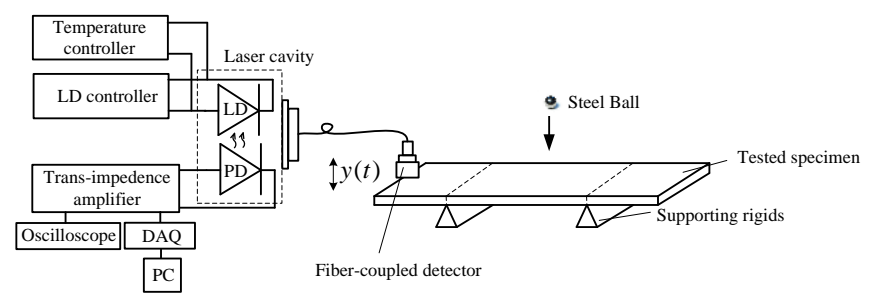

Fig. 1. Schematic FCSM measuring system

$$
\begin{gathered}
\phi_{F}(t)=\phi_{0}(t)-C \sin \left[\phi_{F}(t)+\arctan (\alpha)\right] \\
G(t)=\cos \left(\phi_{F}(t)\right) \\
P(t)=P_{0}[1+m G(t)]
\end{gathered}
$$


Hence, from (2) - (4), $P(t)$ can be recognized as the output of the measuring system, while $y(t)$ is thought as the input. By applying FFT on $P(t), f_{R O}$ can be retrieved by the first peak from the amplitude spectrum of signal from $y(t)$. In the following, we will present how to perform the actual test for Young's modulus measurement.

\section{EXPERIMENTS}

The experiments were conducted on an aluminum alloy 6061 specimen with $L=132.43 \mathrm{~mm}, \quad b=12.24 \mathrm{~mm}, h=$ $2.00 \mathrm{~mm}$ and $m=8.70 \mathrm{~g}$.

Step 1: Install the LD (L785P090, 785nm, 90mW) onto a laser mount; set the bias current as $52.5 \mathrm{~mA}$; then chose the right lens that collimates the light or focus at the point where the fibre-port collimator is located, so that the light intensity can be coupled as much into the fibre as possible. An optical power meter can be used to maximize the laser power.

Step 2: A suitable end collimator for coupling the light is adopted to make sure the light is collimated at the other end of the fibre, and its light intensity is big enough so that light intensity of the feedback of the laser from the specimen can be detected by PD through the fibre.

Step 3: Place the steel ball on the up end of the guided tube and release it. As a result, the specimen is stimulated into vibration.

For each specimen, step 4 was repeated for 10 times. Thus 10 pieces of SMI signals $P(t)$ were collected and the corresponding spectrums are calculated by applying FFT. For illustration, we show one of the experimental results in Fig. 2.

The sampling rates were all set as $200 \mathrm{KHz}$ during the experiments. The data length for each piece of signal is 200,000 points. Hence, the resolution of each spectrum can

TABLE I

RESULTS OF MEASUREMENT AND CALCULATIONS

\begin{tabular}{|c|c|c|}
\hline \multirow{2}{*}{ Simes(N) } & \multicolumn{2}{|c|}{ Aluminum 6061 } \\
\cline { 2 - 3 } & $f_{R O}(\mathrm{~Hz})$ & $E(\mathrm{GPa})$ \\
\hline 1 & 599 & 70.18 \\
\cline { 2 - 3 } 3 & 598 & 69.95 \\
\cline { 2 - 3 } 4 & 599 & 70.18 \\
\cline { 2 - 3 } 5 & 598 & 69.95 \\
\cline { 2 - 3 } 6 & 597 & 69.72 \\
\cline { 2 - 3 } 7 & 598 & 69.95 \\
\cline { 2 - 3 } 8 & 599 & 70.18 \\
\cline { 2 - 3 } 9 & 598 & 69.95 \\
\cline { 2 - 3 } 10 & 599 & 70.18 \\
\cline { 2 - 3 } & 598 & 69.95 \\
\hline Mean $(\mu)$ & 598 & 70.02 \\
\hline $\begin{array}{c}\text { Standard } \\
\text { deviation }(\sigma)\end{array}$ & 0.68 & 0.16 \\
\hline
\end{tabular}
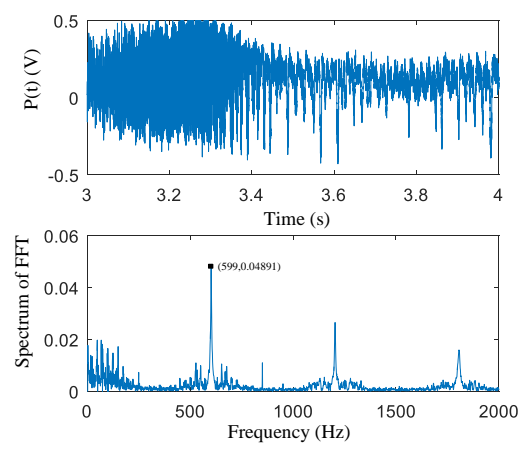

Fig. 2. Experimental results of aluminum 6061

reach to $1 \mathrm{~Hz}$. From the spectrums in Fig. 3, the first peak $(599 \mathrm{~Hz})$ is detected as the fundamental resonant frequency $f_{R o}$. It is characterized as the highest peak in the spectrum. The Measurement details of fundamental resonant frequency $f_{\text {ro }}$ are shown in Table I.

From the standard deviation given in Table $I$, the measurement accuracy for $f_{R o}$ and $E$ are respectively calculated by $(\sigma / \mu) \%$ as $0.68 \%$ and $0.16 \%$. The Young's modulus is $70.02 \mathrm{GPa}$, which is fallen in the rages of 69 $72 \mathrm{GPa}$ reported in literatures[4].

\section{CONCLUSION}

An FCSM laser measuring system is established for Young's modulus measurement based on impulse excitation technique. Experiment results show that high accuracy of measurement can be achieved with high repeatability. The new FCSM measuring system also presents its advantage of compactness and potential application for multi-point detection in the future.

\section{REFERENCES}

[1] S. Tognana, W. Salgueiro, A. Somoza, and A. Marzocca, "Measurement of the Young's modulus in particulate epoxy composites using the impulse excitation technique," Materials Science and Engineering: A, 2010 vol. 527(18): pp. 4619-4623.

[2] K. Lin, et al. System implementation of self-mixing interferometry technique-based measurement on Young's modulus. in SPIE/COS Photonics Asia. 2014. International Society for Optics and Photonics.

[3] ASTM Standard E 1876 - 01, "Standard Test Method for Dynamic Young's Modulus, Shear Modulus, and Poisson's Ratio by Impulse Excitation of Vibration," ASTM., West Conshohocken,, 2005.

[4] R.M. Digilov and H. Abramovich, "Flexural Vibration Test of a Beam Elastically Restrained at One End: A New Approach for Young's Modulus Determination," Advances in Materials Science and Engineering, 2013, vol. 2013. 